\title{
Double lung transplantation for end-stage Kartagener syndrome: a case report and literature review
}

\author{
Bo Wang ${ }^{1}$, Xinghua Zhang ${ }^{1}$, Wenyang Jiang ${ }^{1}$, Jie Huang ${ }^{1}$, Jingyu Chen ${ }^{2}$, Daniel Kreisel ${ }^{3}$, \\ Jose Luis J. Danguilan ${ }^{4}$, Michael Hsin ${ }^{5}$, Huiqing Lin ${ }^{1}$ \\ ${ }^{1}$ Department of Thoracic Surgery, Renmin Hospital of Wuhan University, Wuhan 430060, China; ${ }^{2}$ Department of Thoracic Surgery, Nanjing \\ Medical University, Affiliated Wuxi People's Hospital, Wuxi 214000, China; ${ }^{3}$ Division of Cardiothoracic Surgery, Washington University in St. \\ Louis, Saint Louis, Missouri, USA; ${ }^{4}$ Department of Thoracic Surgery and Anesthesia, Lung Center of the Philippines, Quezon City, Philippines; \\ ${ }^{5}$ Department of Cardiothoracic Surgery, Queen Mary Hospital, High West, Hong Kong, China \\ Correspondence to: Huiqing Lin. Department of Thoracic Surgery, Renmin Hospital of Wuhan University, Wuhan 430060, China. \\ Email: huiqing.lin@whu.edu.cn.
}

\begin{abstract}
Kartagener syndrome (KS) is an autosomal recessive disorder characterized by situs inversus, paranasal sinusitis and bronchiectasis. We report the successful use of double lung transplant (DLTx) to treat end-stage KS. A 49-year-old Han woman was admitted to Renmin Hospital (Wuhan University, China) in September 2017 with a $\geq 15$ year history of chronic productive cough that had worsened during the past year. Clinical examination and imaging investigations revealed respiratory failure and situs inversus consistent with KS. The patient was successfully treated with DLTx involving bilateral bronchial anastomoses. DLTx is a feasible treatment option for end-stage KS.
\end{abstract}

Keywords: Kartagener syndrome; lung transplantation; primary ciliary dyskinesia; situs inversus

Submitted May 08, 2019. Accepted for publication Feb 04, 2020.

doi: $10.21037 /$ jtd.2020.02.28

View this article at: http://dx.doi.org/10.21037/jtd.2020.02.28

\section{Introduction}

Kartagener syndrome (KS) is a rare autosomal recessive disorder with an incidence of 1 per 20,000-30,000 live births (1). KS is a primary ciliary dyskinesia (PCD) characterized by situs inversus, paranasal sinusitis and bronchiectasis (2). Clinical manifestations of KS usually present during childhood and include chronic rhinitis due to pansinusitis, cough, wheeze and shortness of breath due to recurrent respiratory tract infections and bronchiectasis, and hearing problems due to otitis media $(3,4)$. Recommended diagnostic investigations for KS include nasal nitric oxide measurement, ciliary biopsy with electron microscopy, functional ciliary beat/waveform analysis with high-speed videomicroscopy, and genetic testing (3).

Routine treatment of KS involves daily chest physiotherapy for airway clearance, antibiotics for acute respiratory exacerbations and vaccinations. Therapies considered on a case-by-case basis include chronic suppressive antibiotics, inhaled hyperosmolar agents (to promote cough clearance) and bronchodilators (3). Lung transplantation (LTx) is an option for end-stage KS, which results in respiratory insufficiency (5).

Here, we report the successful use of double LTx (DLTx) to treat a 49-year-old patient with end-stage KS.

\section{Case presentation}

A 49-year-old Han woman was admitted to Renmin Hospital of Wuhan University (Hubei, China) on September 8,2017 . She had a $\geq 15$ year history of chronic bronchiectasis with chest discomfort, productive cough and expectoration and had been treated with intranasal steroids and antibiotics for suspected diagnoses of chronic sinusitis and recurrent pneumonia. During the past year, her cough had exacerbated with the production of copious purulent sputum, and she underwent an 8-month course of therapy for suspected smear-negative pulmonary tuberculosis. The patient was a homemaker living in the countryside and had 

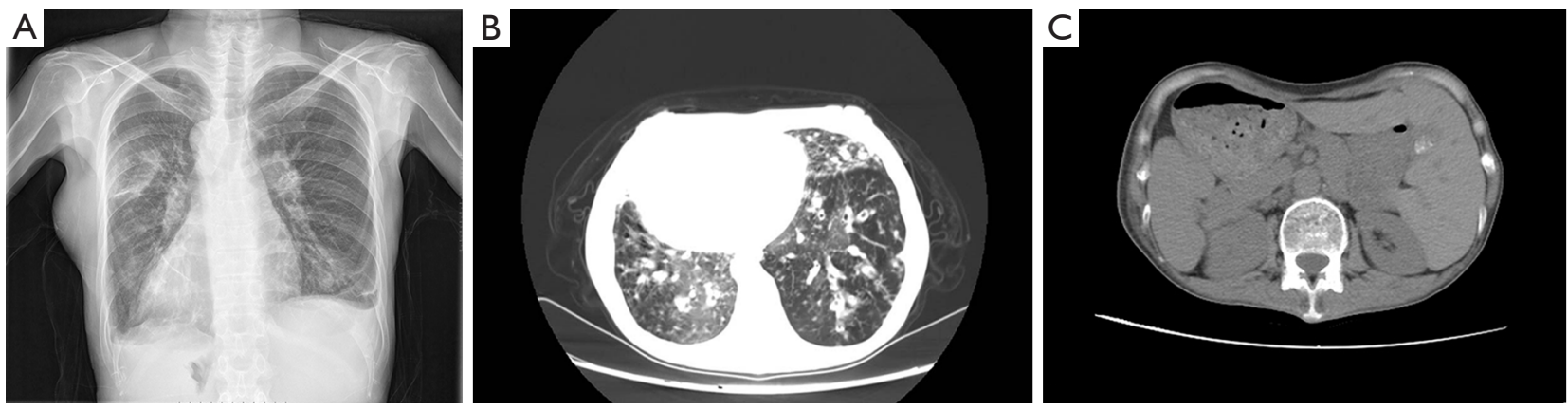

Figure 1 Imaging shows situs inversus and lung infection. (A) Anteroposterior chest X-ray showing dextrocardia; (B) chest CT showing bilateral lower lung bronchiectasis with infection; (C) abdominal CT showing situs inversus.

never smoked cigarettes. There were no similar illnesses in her family. This study was conducted in full accordance with ethical principles (World Medical Association Declaration of Helsinki, and the Declaration of Istanbul) and was approved by ethics committee of Renmin Hospital of Wuhan University.

Physical examination revealed the following: weight, $54 \mathrm{~kg}$; body mass index, $18.6 \mathrm{~kg} / \mathrm{m}^{2}$; blood pressure, 91/60 $\mathrm{mmHg}$; pulse rate, 109 beats/minute; respiratory rate, 20 breaths/minute; temperature, $36.8^{\circ} \mathrm{C}$; and arterial oxygen saturation (room air), $86 \%$. There were coarse crackles and scattered rhonchi over both basal lung fields. The cardiac apex beat was palpable in the right fifth intercostal space along the midclavicular line, and heart sounds were best heard on the right side of the chest. Abdominal examination revealed a tympanitic note on percussion and no sign of fluid collection. The patient did not have any dyskinesia. Nervous system examination was unremarkable.

Laboratory investigations revealed normal values for hemoglobin (105 g/L), leukocyte count $\left(5.97 \times 10^{9} / \mathrm{L} ; 74 \%\right.$ granulocytes and $16.4 \%$ lymphocytes), platelet count $\left(130 \times 10^{9} / \mathrm{L}\right)$, and serum biochemistry tests. The patient had chronic airway colonization with Gram-negative bacteria but was negative for human immunodeficiency virus and mycobacterium tuberculosis (sputum test for acid fast bacilli). Arterial blood gas analysis revealed oxygendependent respiratory failure and hypercapnia $\left(\mathrm{PO}_{2}\right.$, $52 \mathrm{mmHg} ; \mathrm{PCO}_{2}, 82 \mathrm{mmHg}$; base excess, $19 \mathrm{mmol} / \mathrm{L}$ ) that required 24-hour oxygen support.

Anteroposterior chest radiography revealed a right-sided cardiac apex and aortic arch (Figure 1A). Echocardiography demonstrated dextrocardia and a pulmonary artery systolic pressure of $49 \mathrm{mmHg}$. High-resolution computed tomography (CT) of the chest showed bronchiectasis and fibrotic bands in both lower lung fields with no obvious lymph nodes in the mediastinum (Figure 1B). Abdominal CT showed a left-sided liver and inferior vena cava and a right-sided spleen, suggesting situs inversus (Figure 1C). Exome sequencing revealed mutations in the HEATR, DNAH1, DNAH5, DNAHI1, DNAH9, DNAI2 and $D N A A F 3$ genes (Table 1). A diagnosis of $\mathrm{KS}$ was made based on the clinical findings $(4,6)$.

The patient's symptoms were partially relieved after several courses of antibiotics, oral steroids and bronchodilators but recurred after therapy was discontinued. The patient fulfilled the criteria for DLTx (5) and so was evaluated at our hospital for LTx. The measured/predicted recipient total lung capacity was 3.78/3.51 L (ratio: 1.08). After 6 months on the waiting list (March 31, 2018), a suitable donor was allocated by Chinese Donor Allocation System COTAS with the following characteristics: 32-yearold male; weight, $58 \mathrm{~kg}$; height, $156 \mathrm{~cm}$; matching blood type; clear chest radiograph; no history of smoking, brain trauma or malignancy; and $\mathrm{PaO}_{2} / \mathrm{FiO}_{2}$ ratio of $563 \mathrm{mmHg}$.

The planned surgery was bilateral lung transplantation via bilateral anterolateral thoracotomy without the use of central extracorporeal membrane oxygenation (because our patient had a pulmonary artery systolic pressure of $28 \mathrm{mmHg}$ ). Left lung transplantation was performed first with an ischemic time of 132 min followed by right lung transplantation with an ischemic time of $224 \mathrm{~min}$. An inadequate donor left atrial cuff due to technical errors at the time of procurement was evident during right lung transplantation. A partial patch repair with donor aorta remnant was performed, and the reconstruction was carried out using a 5-0 polypropylene running suture. Care was taken not to stretch or twist the pulmonary venous anastomosis (Figure $2 A, B$ ). All vascular anastomoses were carefully inspected to detect any tension, 
Table 1 Exome sequencing results

\begin{tabular}{ll}
\hline Gene name & AAChange.refGene \\
\hline HEATR1 & HEATR1:NM_018072:exon8:c.A1043G:p.H348R \\
DNAH1 & DNAH1:NM_015512:exon72:c.A11495G:p.H3832R \\
DNAH5 & DNAH5:NM_001369:exon13:c.A1672G:p.T558A \\
DNAH5 & DNAH5:NM_001369:exon2:c.G71A:p.G24E \\
DNAH5 & DNAH5:NM_001369:exonl:c.T36G:p.H12Q \\
DNAH11 & DNAH11:NM_001277115:exon64:c.G10399A:p.A3467T \\
DNAH9 & DNAH9:NM_001372:exon32:c.G6485A:p.R2162K \\
DNAH9 & DNAH9:NM_004662:exon14:c.G2058A:p.M686I,DNAH9:NM_001372:exon68: c.G13122A;p.M4374I \\
DNAI2 & DNAI2:NM_0011728 10:exon12:c.G1636A:p.A546T,DNAI2:NM_023036:exon12:c.G1672A:pA558T \\
DNAAF3 & DNAAF3:NM_001256714:exon10:c.G1294A:p.D432N, DNAAF3:NM_001256715:exon10:c.G1093A:p.D365N, \\
& DNAAF3:NM_001256716:exon10:c.G93 1A:p.D31IN, DNAAF3:NM_178837:exon10:c.G1234A:p.D412N \\
\hline
\end{tabular}
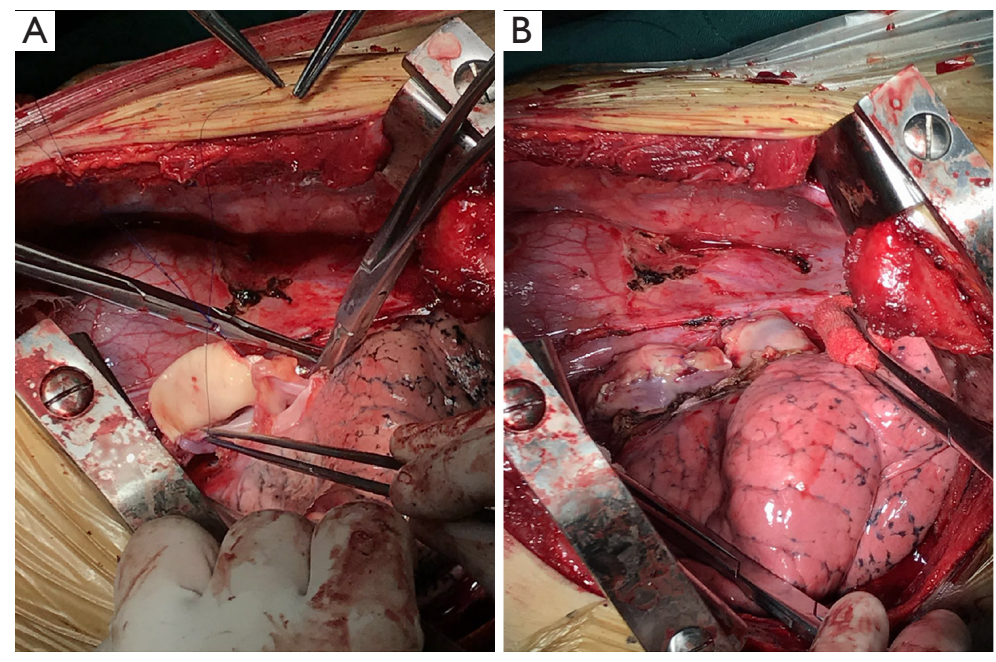

Figure 2 The procedures of repairing and reconstructing an inadequate left cuff during lung transplantation. (A) A donor aorta patch was used to repair and reconstruct an inadequate left cuff during surgery; (B) left atrium after intraoperative anastomoses.

kinking, narrowing or bleeding.

The patient was discharged to the intensive care unit and extubated on postoperative day 2. For immunosuppression, she received a standard triple regimen for 3 days (tacrolimus $0.1 \mathrm{mg} / \mathrm{kg}$ PO bid, mycophenolate mofetil $1 \mathrm{~g}$ IV bid, and methylprednisolone $0.5 \mathrm{mg} / \mathrm{kg}$ IV qd) followed by oral prednisone from day $4(0.5 \mathrm{mg} / \mathrm{kg}$ PO qd, tapering down by $5 \mathrm{mg} /$ week to $0.25 \mathrm{mg} / \mathrm{kg} /$ day). Pseudomonas aeruginosa and methicillin-resistant Staphylococcus aureus, which were isolated from bronchoalveolar secretions obtained during bronchoscopy, were treated according to the antimicrobial resistance data and current guidelines. The patient recovered well and was discharged on postoperative day 18 .

The patient was followed-up 21 months after transplantation. Her symptoms of cough and purulent sputum were relieved. At the last follow-up, the patient was doing well and was able to engage in her usual daily activities. Bronchoscopy was done 18 months after transplantation, which demonstrated a clear anastomotic airway without excessive granulation tissue, bronchomalacia, airway stenosis or fistula, and the anastomotic site showed no evidence of mucosal necrosis or sloughing (Figure $3 A, B, C$ ). 


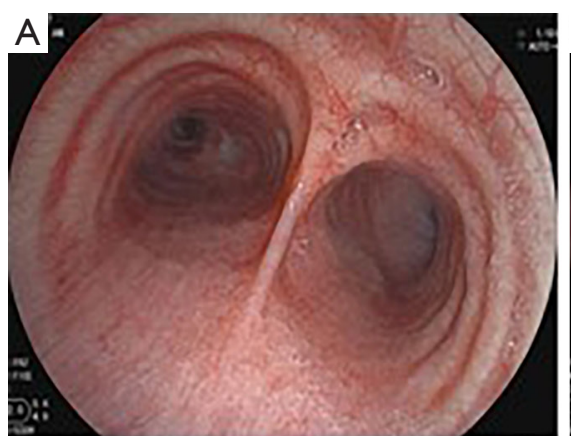

Tracheal carina

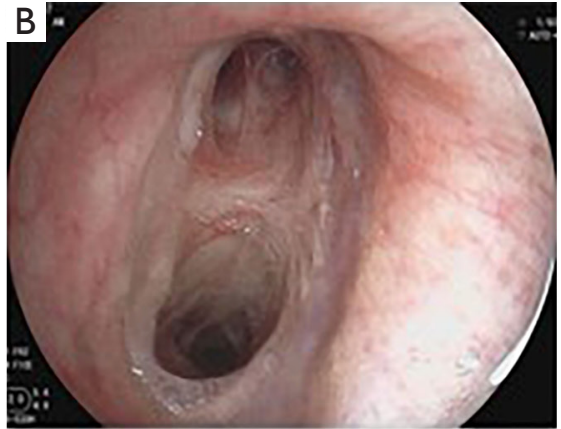

Right anastomotic airway

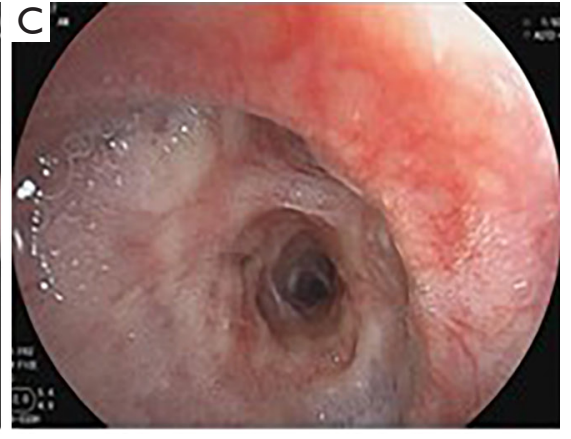

Left anastomotic airway

Figure 3 Bronchoscopy demonstrated a clear anastomotic airway.

Based on the physical examination and investigations, this patient was diagnosed to suffer from end-stage KS. Antibiotics, oral steroids and bronchodilators were administered which partially relieved the patient's symptoms. However, because of the end-stage bronchiectasis secondary to KS, double lung transplantation (DLTx) was eventually performed with satisfactory outcomes up to 21 months follow-up.

\section{IMDT discussion}

\section{Discussion among physicians from the Wuban University of Renmin Hospital}

Ciliary dysfunction is the underlying cause of KS (2). Impaired ciliary motility during embryogenesis predisposes to left-right laterality defects such as situs solitus (dextrocardia only) or situs inversus totalis (with transpositions of thoracic and abdominal organs). In addition to detrimental effects in the respiratory system, impaired ciliary motility can cause infertility secondary to sperm tail and fallopian tube dysfunction $(3,6)$.

There are no curative treatments for KS. The main therapeutic measures for early-stage KS are daily chest physiotherapy, inhaled bronchodilators, vaccinations and antibiotics. For end-stage KS, LTx may be useful. The first heart and lung transplantation for KS (a 25-year-old woman with a history of recurrent pulmonary infection) was described in 1992 (7). Successful left LTx for KS was described 2 years later (8), but only a few additional case reports have been published since then, emphasizing the challenges of LTx in patients with KS. Up to 2016, 28 patients with PCD or KS had undergone LTx in the USA, and their 1-year survival was comparable to that of patients with idiopathic pulmonary fibrosis, cystic fibrosis or chronic obstructive pulmonary disease (9). Surgical management of KS has been described for 2 cases in China, one treated with lobectomy and the other with LTx (10).

Very few case reports have described DLTx in patients with KS. The configuration and length of the main stem bronchi are inverted in patients with situs inversus, but no technical modifications for the different anatomic relationships were required in our case. The midline position of the left atrium and pulmonary artery allowed anastomoses with the recipient's structures to be made as in patients with situs solitus. Previous publications have emphasized the potential anatomical conflict between the donor's right lower lobe and the recipient's dextrocardia, although lung volume reduction surgery was not necessary $(8,11,12)$. However, in a previous case in China, right lower lobectomy was required during LTx because the volume of the right donor lung was larger than that of the recipient (10). In our patient, different bronchial angulation was not an obstacle, and airway continuity was reestablished with end-to-end anastomoses without the need for lung volume reduction (Figure 4).

There are some questions for further discussion.

\section{Question 1: is there any difference between KS and normal patients in the procedure of DLTx? Expert opinion 1: Dr. Daniel Kreisel}

We have recently reported the case of double lung transplantation in a patient with Kartagener syndrome and described technical modifications to account for the anatomical variations in these patients (13). Since these patients have a long right mainstem bronchus and a short left mainstem bronchus, a right-sided double lumen endotracheal tube was used for our procedure that was placed in the anatomic right mainstem bronchus. Some technical modifications were required for the vascular anastomoses. The recipient left pulmonary artery was 

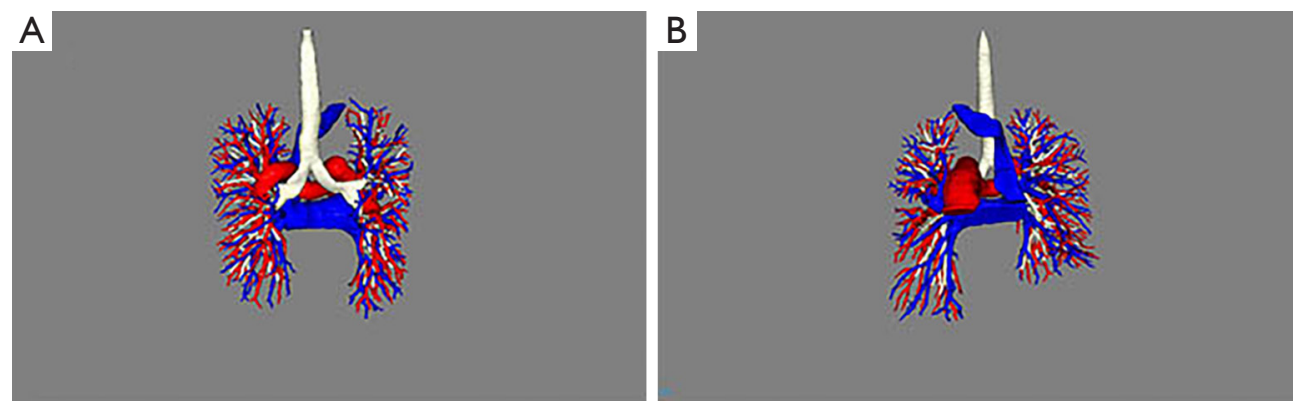

Figure 4 Image of the reconstructed pulmonary vessels and bronchi obtained 5 months after surgery.

located anterior to the bronchus while the donor left pulmonary artery was located superior to the bronchus. An end-to-end anastomosis between donor and recipient pulmonary arteries was facilitated by mobilizing the recipient pulmonary artery and transposing it to an epibronchial position. In addition, we beveled the donor pulmonary artery to optimize the orientation of the end-toend anastomosis. We found that the right donor pulmonary artery needed to be adequately mobilized to account for a relatively short right pulmonary artery in the recipient. In our experience, no technical modifications were required for the bronchial or venous anastomoses. While reports exist that the size of the right lung graft needs to be reduced due to space limitations of the right chest cavity in the recipient, we did not find this necessary in our case.

\section{Expert opinion 2: Dr. Fose Luis 7. Danguilan}

Yes, there is a difference in double lung transplantation in patients with Kartagener's syndrome specially in those with anatomical variations like pulmonary situs inversus and dextrocardia (13). A right-sided double-lumen endotracheal tube was useful in the performance of pneumonectomy of the anatomic left lung with division of the short recipient left main bronchus $(8,14)$.

A right lower lobectomy was required after implantation due to the right-sided heart interfering with lung expansion (14) although no technical modifications for the different anatomic relationships were necessary in a DLTx performed in a 46-year-old KS patient and discrepancies of the bronchial lumina were easily overcome (12).

Furthermore, a detailed preoperative anatomical study using multidetector row computed tomography (MDCT) with $3 \mathrm{D}$ reconstruction should be undertaken by the lung transplant team especially in patients with KS considering variations in anatomy (15).

\section{Expert opinion 3: Dr. Michael Hsin}

Since 1995, the Queen Mary Hospital Cardiothoracic Surgery
Hospital Lung Transplant Program has performed two cases of double lung transplant for Kartagener's syndrome, with one case in 2015 and the other case in 2019. The case from 2015 was performed entirely with intraoperative ECMO support. For the more recent case, the left donor lung implantation was done without ECMO. When it came to the right side donor lung implantation, to achieve the necessary exposure the amount of retraction on the heart (because of dextrocardia) required intraoperative ECMO support.

For the case done in 2015, because of excessive size mismatch, lung volume reduction was required from the right middle lobe and the lingula. No lung volume reduction was needed for the case done in 2019. Similar to the current case report from the Wuhan group, there were no special modifications of the surgical technique for any of the anastomoses in either of the two cases from the Queen Mary Hospital. Both cases had uneventful operations and early post-operative recovery.

A recent case report from the St Louis group (13) did focus on the technical considerations in Kartagener's syndrome. They pointed out the following:

(I) An initial attempt to use a standard left-sided double lumen endotracheal tube was unsatisfactory, and had to switch to using a right-sided double lumen endotracheal tube;

(II) For the recipient's left pulmonary artery (PA) anastomosis, they noted that the recipient's PA had a prebronchial position (i.e., anterior to the bronchus), whereas the donor PA had an epibronchial (superior to bronchus) location. The authors explained that they had to adequately mobilize the PA for both donor and recipient, and they had to divide the PA at a bevel for size and orientation matching.

Another earlier report from the Brigham and Women's hospital described the use of an end-to side anastomosis to deal with the issue of left PA anastomosis (8). In order to 
avoid distortion of the PA, the recipient left PA was divided just beyond the origin of the truncus anterior, and the stump was oversewn. The donor PA was the anastomosed to the recipient at the origin of the truncus anterior. Although no kinking of the PA was encountered, the authors did mention that in postoperative angiogram, they noted variable pressure in the area of the anastomosis, suggesting turbulence of the pulmonary artery blood flow. Indeed, Gauthier et al. (13) commented that they did not use the end-to side anastomosis because of their concern regarding the risk of stasis in the $\mathrm{PA}$ arterial stump of the recipient $\mathrm{PA}$.

\section{Question 2: when the KS patient should consider about} the surgery?

\section{Expert opinion 1: Dr. Daniel Kreisel}

Criteria to refer patients with Kartagener syndrome for lung transplantation generally resemble those for other patients, who suffer from bronchiectatic diseases such as cystic fibrosis. These criteria include forced expiratory volume in 1 second (FEV1) $\leq 25 \%$ predicted, hypercapnia, pulmonary hypertension and frequent exacerbations.

\section{Expert opinion 2: Dr. Fose Luis 7. Danguilan}

As with other indications for lung transplantation, the patient with KS should be considered for transplantation if they have end-stage lung disease brought about by repeated infections and retained airway secretions from bronchiectasis for which maximal medical management and conventional lung resections would be futile. DLTx has been described for generalized bronchiectasis (16).

\section{Expert opinion 3: Dr. Michael Hsin}

Patients with Kartagener's syndrome have primary ciliary dyskinesia (PCD), and the accumulation of airway secretions cause recurrent infections, eventually manifesting as bronchiectasis. In a minority of patients, this progresses to end-stage bronchiectasis. As pointed by the authors of this manuscript, there are only a handful of publications regarding lung transplantation in KS. Indeed, even for non-cystic fibrosis bronchiectasis, there is not a lot of published data available. However, the indicators of endstage lung disease in general bronchiectasis as outlined by Hayes et al can be useful as points of reference (9). These are: post-bronchodilator FEV1 $<30 \%$; exacerbation of pulmonary disease needing intensive care stay; increasing frequency of exacerbation needing intravenous antibiotics; refractory and/or recurrent pneumothorax; recurrent hemoptysis not controlled by embolization; other factors to consider include hypercarbia, rapidly declining lung function, and resting hypoxemia.
Question 3: what about the survival outcomes for the KS patients with DLTx?

\section{Expert opinion 1: Dr. Daniel Kreisel}

Lung transplantation for Kartagener syndrome is rare. A review of the United Network for Organ Sharing registry showed that 12 bilateral lung transplants have been performed between 1987 and 2015 (9). The 1-year and 5 -year survival rates after bilateral lung transplantation for Kartagener syndrome patients were $74 \%$ and $65 \%$, respectively. Of note, no statistically significant differences existed between the survival rates after lung transplantation for Kartagener syndrome and lung transplantation for other conditions such as idiopathic pulmonary fibrosis, cystic fibrosis or chronic obstructive pulmonary disease.

\section{Expert opinion 2: Dr. Fose Luis F. Danguilan}

Since only a few patients with KS have undergone lung transplantation since 1987 (9), it would be difficult to accurately predict factors leading to long-term survival in this subset of patients. However, it is safe to say that lung transplantation markedly improves lung function and exercise tolerance but post-transplantation survival depends on the development of chronic lung allograft dysfunction (CLAD) (17).

\section{Expert opinion 3: Dr. Michael Hsin}

As stated in this manuscript, the study from Hayes et al. which queried the UNOS database between 1987-2015 on Kartagener's syndrome, the long term outcome after LTx is comparable to the general LTx population (9). Specifically, when compared to idiopathic pulmonary fibrosis (IPF), cystic fibrosis (CF) and chronic obstructive pulmonary disease (COPD) recipients, using Cox proportional hazards regression, there were no differences in survival between KS group and these groups. Nor was there any significant survival advantage between KS group and these groups at one- year post lung transplant (9). Interestingly, the authors also looked at patients with PCD (impaired mucociliary clearance without situs inversus and chronic sinusitis). Whilst comparisons of overall survival between PCD and IPF, CF and COPD showed no difference, at one- year post transplant, there was survival advantage for $\mathrm{CF}$ and COPD relative to $\mathrm{PCD}$.

\section{Conclusions}

In summary, we have reported the successful use of DLTx to treat a patient with end-stage KS and respiratory insufficiency. DLTx may be a feasible therapeutic option for controlling symptoms and achieving long term survival in patients with end-stage KS. 


\section{Acknowledgments}

The authors thank Prof. Handong Zou and Prof. Guozhong Chen for their academic and technical support.

Funding: This study was supported by the National Natural Science Foundation of China (81800343).

\section{Footnote}

Conflicts of Interest: All authors have completed the ICMJE uniform disclosure form (available at http://dx.doi. org/10.21037/jtd.2020.02.28). DK reports grants and personal fees from Compass Therapeutics, outside the submitted work; In addition, DK has a pending US patent entitled "Compositions and methods for detecting CCR2 receptors" (application number. The other authors have no conflicts of interest to declare.

Ethical Statement: The authors are accountable for all aspects of the work in ensuring that questions related to the accuracy or integrity of any part of the work are appropriately investigated and resolved.

Open Access Statement: This is an Open Access article distributed in accordance with the Creative Commons Attribution-NonCommercial-NoDerivs 4.0 International License (CC BY-NC-ND 4.0), which permits the noncommercial replication and distribution of the article with the strict proviso that no changes or edits are made and the original work is properly cited (including links to both the formal publication through the relevant DOI and the license). See: https://creativecommons.org/licenses/by-nc-nd/4.0/.

\section{References}

1. Abbasi MA, Suleman A, Ahmed N, et al. Kartagener syndrome: a not so rare phenomenon. J Ayub Med Coll Abbottabad 2014;26:598-601.

2. Knowles MR, Zariwala M, Leigh M. Primary Ciliary Dyskinesia. Clin Chest Med 2016;37:449-61.

3. Shapiro AJ, Zariwala MA, Ferkol T, et al. Diagnosis, monitoring, and treatment of primary ciliary dyskinesia: PCD foundation consensus recommendations based on state of the art review. Pediatr Pulmonol 2016;51:115-32.

4. Mishra M, Kumar N, Jaiswal A, et al. Kartagener's syndrome: A case series. Lung India 2012;29:366-9.

5. Leigh MW, Pittman JE, Carson JL, et al. Clinical and genetic aspects of primary ciliary dyskinesia/Kartagener syndrome. Genet Med 2009;11:473-87.
6. Ceccaldi PF, Carre-Pigeon F, Youinou Y, et al.

Kartagener's syndrome and infertility: observation, diagnosis and treatment. J Gynecol Obstet Biol Reprod (Paris) 2004;33:192-4.

7. Miralles A, Muneretto C, Gandjbakhch I, et al. Heart-lung transplantation in situs inversus. A case report in a patient with Kartagener's syndrome. J Thorac Cardiovasc Surg 1992;103:307-13.

8. Mentzer SJ, Aranki SF, Reilly JJ, et al. Single-lung transplantation in situs inversus. Ann Thorac Surg 1994;58:1176-8.

9. Hayes D Jr, Reynolds SD, Tumin D. Outcomes of lung transplantation for primary ciliary dyskinesia and Kartagener syndrome. J Heart Lung Transplant 2016;35:1377-8.

10. He YJ, Chen R, Mao WJ, et al. Surgical Therapy for two Cases of Complete Kartagener Syndrome and Effect Evaluation. Progress in Modern Biomedicine 2016;16:6060-4.

11. Brioude G, D'Journo XB, Reynaud-Gaubert M, et al. Bronchial fistula after lobar size reduction for bilateral lung transplantation in Kartagener's syndrome: a surgical challenge. Interact Cardiovasc Thorac Surg 2013;17:184-6.

12. Graeter T, Schafers HJ, Wahlers T, et al. Lung transplantation in Kartagener's syndrome. J Heart Lung Transplant 1994;13:724-6.

13. Gauthier JM, Takahashi T, Bierhals AJ, et al. Technical Considerations for Lung Transplantation in Kartagener's Syndrome. Ann Thorac Surg 2019;107:e337-9.

14. Macchiarini P, Chapelier A, Vouhe P, et al. Double lung transplantation in situs inversus with Kartagener's syndrome. Paris-Sud University Lung Transplant Group. J Thorac Cardiovasc Surg 1994;108:86-91.

15. Schertler T, Lardinois D, Boehm T, et al. Lung transplantation in Kartagener syndrome and situs inversus: potential of multidetector row computed tomography and three-dimensional postprocessing. J Thorac Cardiovasc Surg 2007;134:814-5.

16. Lung transplantation. Report of the ATS workshop on lung transplantation. American Thoracic Society, Medical Section of the American Lung Association. Am Rev Respir Dis 1993;147:772-6.

17. Thabut G, Mal H. Outcomes after lung transplantation. J Thorac Dis 2017;9:2684-91.

Cite this article as: Wang B, Zhang $\mathrm{X}$, Jiang W, Huang J, Chen J, Kreisel D, Danguilan JLJ, Hsin M, Lin H. Double lung transplantation for end-stage Kartagener syndrome: a case report and literature review. J Thorac Dis 2020;12(4):1588-1594. doi: $10.21037 /$ jtd.2020.02.28 